\title{
GC-FID and NMR Spectroscopic Studies on Gamma Irradiated Walnut Lipids
}

\author{
Vassilia J. Sinanoglou, ${ }^{1}$ Irini F. Strati, ${ }^{1}$ Katerina Kokkotou, ${ }^{2}$ Dimitra Lantzouraki, \\ Constantinos Makris, ${ }^{1}$ and Panagiotis Zoumpoulakis ${ }^{2}$ \\ ${ }^{1}$ Instrumental Food Analysis Laboratory, Department of Food Technology, Technological Educational Institution of Athens, \\ Ag. Spyridonos, 12210 Egaleo, Greece \\ ${ }^{2}$ Institute of Biology, Medicinal Chemistry and Biotechnology, National Hellenic Research Foundation, \\ 48 Vas. Constantinou Avenue, 11635 Athens, Greece
}

Correspondence should be addressed to Panagiotis Zoumpoulakis; pzoump@eie.gr

Received 31 October 2014; Accepted 8 March 2015

Academic Editor: Feride Severcan

Copyright (C) 2015 Vassilia J. Sinanoglou et al. This is an open access article distributed under the Creative Commons Attribution License, which permits unrestricted use, distribution, and reproduction in any medium, provided the original work is properly cited.

Walnuts have an excellent fatty acid profile, beneficial for coronary heart diseases. A diet rich in walnuts has shown to decrease the total and LDL cholesterol levels as well as lipoprotein levels. In this study, the effects of different doses of $\gamma$-irradiation and different packaging conditions on proximate composition and fatty acid profile of walnuts (Juglans regia L.) were investigated merging data from different spectroscopic techniques. Walnuts moisture, ash, fat, and protein content as well as fatty acid profile were evaluated immediately after irradiation. GC-FID results showed that SFA increased and MUFA and PUFA decreased with the increase of irradiation dose. Moreover, MUFA/SFA and PUFA/SFA ratios decreased $(P<0.05)$ compared to control samples. Furthermore, NMR spectroscopy was implemented to examine possible discrimination patterns based on irradiation dose and packaging. This approach revealed the role of PUFA decrease with the parallel increase of irradiation dose while indicating the protective role of vacuum and MAP compared to air packaging. In conclusion, at irradiation doses of up to $5 \mathrm{kGy}$, the walnuts retained the nutritional benefits of its fatty acids, in particular MUFA and PUFA. Concerning the different types of packaging, greater stability in the nuts was observed using MAP packaging.

\section{Introduction}

Walnuts (nux juglandes) are harvested from walnut trees (Juglans regia) and are considered as one of the most popular nuts worldwide, either consumed raw, toasted or pickled or as food ingredient. Over 30 varieties of walnut trees are currently harvested that have been developed for various characteristics including pest tolerance, early/late harvest, and shell thickness [1].

Walnuts contain health benefiting nutrients, minerals, antioxidants, and vitamins that are essential for optimum health. Due to their high polyunsaturated fatty acids (PUFA, predominantly linoleic and $\alpha$-linolenic acid), their fatty acid composition is unique compared to other nuts, which contain mostly monounsaturated fatty acids (MUFA) [2]. Additionally, Yang et al. [3] reported that walnuts had the highest flavonoid content, followed by pecans, peanuts, pistachios, cashews, almonds, Brazil nuts, pine nuts, macadamia nuts, and hazelnuts. Evidence from epidemiologic and intervention studies as well as clinical trials showed that walnut consumption had favorable effects on serum lipid levels in humans, such as lowering low-density lipoprotein (LDL), raising high-density lipoprotein (HDL), and reducing total serum triacylglycerol levels, all of which reduce the likelihood of suffering from a cardiovascular event $[4,5]$. Lately, walnuts have been found to improve memory deficits in transgenic mouse model of Alzheimer's disease [6].

Untreated walnuts may be susceptible to microbial contamination or insect and pest infestation; therefore, processing via $\gamma$-irradiation has been recognized and regulated as an effective technology in order to extend the shelf life of all types of nuts [7]. Although the Food and Agriculture Agency/International Atomic Energy Agency/World 
Health Organization (FAO/IAEA/WHO) joint commission endorsed the radiation treatment of food at established doses of up to $10 \mathrm{kGy}$ [8], recently, some countries have allowed irradiation dose above $10 \mathrm{kGy}$ for decontamination purposes [9].

Walnuts contain high levels of unsaturated fatty acids which are prone to lipid oxidation when irradiated. Irradiation produces free radicals as a result of radiolysis. Additionally, interactions of free radicals with both lipid and protein molecules lead to the formation of numerous oxidation products (aldehydes, esters, ketones, sulfur compounds, etc.) and result in off-flavor changes in irradiated foods $[10,11]$.

Most packaging materials for nuts are composed of polymers that may be susceptible to chemical changes induced by ionizing radiation that are the result of two competing reactions, cross-linking (polymerization), and chain scission (degradation). Radiation-induced cross-linking of polymers dominates vacuum or an inert atmosphere. Chain scission dominates during irradiation of polymers in the presence of oxygen or air. Both reactions are random, generally proportional to dose, and depend on dose rate and the oxygen content of the atmosphere in which the polymer is irradiated $[12,13]$.

Up to now, radiolytic products remain the most prominent markers to detect gamma irradiated food items. Recently, our group has demonstrated that global lipid profile of fat rich food items using nuclear magnetic resonance (NMR) spectroscopy and multivariate statistical analysis may constitute an alternative method for the detection of irradiated specimens even at low irradiation doses $[14,15]$.

The objective of the present study was to assess the effect of $\gamma$-irradiation on proximate composition and fatty acid profile of walnuts irradiated at doses of 5, 10, and $13 \mathrm{kGy}$ under atmospheric air, vacuum, and modified atmosphere $\left(41 \% \mathrm{CO}_{2}-59 \% \mathrm{~N}_{2}\right)$ packaging conditions and to determine the appropriate conditions (irradiation dose and packaging) in order to maintain the superior quality of the nuts.

\section{Materials and Methods}

2.1. Reagents and Standards. Fatty acid methyl esters used as gas chromatography (GC) standards were lauric acid methyl-ester (M-E). cis-5,8,11,14,17-Eicosapentaenoic acid M$\mathrm{E}$ and cis-4,7,10,13,16,19-docosahexaenoic acid M-E (purity $\geq$ 98\%) purchased from Sigma Chemical Co (Sigma-Aldrich Company. UK). Fatty acid methyl esters used as GC-FID standard mixtures were Supelco TM 37 Component FAME Mix C4-C24, 100 mg Neat., and Supelco PUFA No. 1, Marine Source, $100 \mathrm{mg}$ Neat.

Chloroform-d (99.98\%) and $5 \mathrm{~mm}$ Norell UP509 tubes were purchased from Sigma-Aldrich Company, UK, for NMR analyses.

All solvents used for sample preparation were of analytical grade and the solvents used for GC-FID analysis were of HPLC grade from Merck (Darmstadt. Germany). All reagents used were of analytical grade and they were purchased from Mallinckrodt Chemical Works (St. Louis, MO, USA) and from Sigma Chemical Co. (Sigma-Aldrich Company, UK).
2.2. Radiation Process. Samples were purchased from a local super market (all samples selected were of the same lot number). Walnuts (Juglans regia L.) were packed in $50 \mathrm{~g}$ sachets, in atmospheric air, vacuum, and modified atmosphere $(41 \%$ $\mathrm{CO}_{2}-59 \% \mathrm{~N}_{2}$ ). Irradiation was carried out in the Institute of Pharmaceutical Research and Technology (IPRT S.A.), (Mandra Attikis, Greece). For gamma irradiation a ${ }^{60} \mathrm{Co}$ source irradiator was used. The strength of the source was $150 \mathrm{kCi}$, with a dose rate capacity of $1 \mathrm{kGy} / \mathrm{h}$. The samples were irradiated under atmospheric air. The doses applied were $5.0,10.0$, and $13.0 \mathrm{kGy}$. The temperature during the irradiation treatment was maintained at approximately $25^{\circ} \mathrm{C}$ (room temperature). Dosimetry was performed using a Harwell Perspex polymethylmethacrylate Amber 3042 dosimeter (PMMA Instruments, Harwell, UK) placed on the irradiated samples surface. Samples were analysed immediately after irradiation. The irradiation process was repeated twice. Three sachets were used per each dose $(0,5,10$, and $13 \mathrm{kGy})$ and per each package (air, vacuum, and modified atmosphere package (MAP)). Total sample set included 72 samples (with control samples).

2.3. Proximate Analysis. Moisture content was determined using an Electronic Moisture Analyser (KERN MLS 50-3, KERN \& Sohn $\mathrm{GmbH}$ ) at $120^{\circ} \mathrm{C}$ to constant weight. Ash was determined by heating in a muffle furnace at $550^{\circ} \mathrm{C}$ to constant weight [16]. Protein content was determined using Kjeldahl method [17] using a BÜCHI digestion unit (K438) and a BÜCHI distillation unit (K-355) (Hellamco S.A., Athens, Greece).

2.4. Extraction Methodologies. A high energy extraction technique (ultrasound assisted extraction (UAE)) was employed since it provided higher yields in significantly shorter time after exhaustive optimizations [14].

In detail, $5 \mathrm{~g}$ of homogenized walnuts sample and $40 \mathrm{~mL}$ solvent (n-hexane) were placed in a $250 \mathrm{~mL}$ three-neck vessel in ice-bath, maximum temperature $40^{\circ} \mathrm{C}$, and sonicated using Sonics \& Material INC., Vibra-Cell VCX750 (20 kHz, 750 W) ultrasonics processor, equipped with piezoelectric converter and $13 \mathrm{~mm}$ diameter probe fabricated from Titanium alloy Ti-6Al-4V. The amplitude was $80 \%$ and the pulse sonication sequence was $15 \mathrm{~s}$ ON and $5 \mathrm{~s}$ OFF. The extraction mixtures were filtrated to separate liquid from solid matrices using a Buchner funnel and the extracts were concentrated in rotary evaporator. Dry residues were weighted and redissolved in $30 \%$ solvent, containing t-butyl-hydroquinone $(0.1 \%)$ as an antioxidant and finally stored at $4^{\circ} \mathrm{C}$ prior to analysis.

2.5. Gas Chromatography Analysis of Fatty Acid Methyl Esters. Fatty acid methyl esters (FAME) of total lipids (TL) were prepared according to the procedure described by Sinanoglou and Miniadis-Meimaroglou [18]. Both quantitative and qualitative analyses were performed on an Agilent 6890 Series Gas Chromatograph (Agilent Technologies, Palo Alto, CA) equipped with a flame ionization detector, as 
described by Sinanoglou et al. [19]. DB-23 capillary column $(60 \mathrm{~m} \times 0.25 \mathrm{~mm}$ i.d. $0.15 \mu \mathrm{m}$ film) $(50 \%$-cyanopropylmethylpolysiloxane) (Agilent Technologies) was used. The individual FAME were identified by comparing their retention times with those of the authentic standard mixtures. The relative content of fatty acids in the sample was determined according to Sinanoglou et al. [19].

2.6. NMR Spectroscopy. Lipid extracts (10 mg approximately) were dissolved in $0.5 \mathrm{~mL}$ chloroform- $d$ (99.98\%). ${ }^{1} \mathrm{H}$ NMR spectra were recorded on a Varian $600 \mathrm{MHz}$ equipped with a triple resonance probe $\{\mathrm{HCN}\}$. The acquisition parameters were as follows: temperature $25^{\circ} \mathrm{C}$, spectral width (SW) $4807 \mathrm{~Hz}$, number of scans 128 , and $32 \mathrm{~K}$ data points. For quantitative purposes the relaxation delay (RD) was at 8 $\mathrm{s}$ as determined by $\mathrm{T} 1$ measurement. Proton spectra were referenced at the resonant peak of $\mathrm{CDCl}_{3}(7.26 \mathrm{ppm})$. receiver gain was kept constant for all acquisitions.

Spectral processing included zero filling up to $32 \mathrm{~K}$, line broadening $0.5 \mathrm{~Hz}$ before Fourier transformation, and it was performed using MestReNova software. Prior to statistical analysis, all spectra were automatically baseline corrected, binned to $0.002 \mathrm{ppm}$ spectral width, and spectral regions of low significance were excluded from the analysis (such as the solvent region, 7.0-7.50 ppm, the water signal at $1.5 \mathrm{ppm}$ ). Finally, each spectrum was normalized to total spectrum area.

\subsection{Statistical Analysis-Multivariate Data Analysis}

2.7.1. Statistical Analysis. All data concerning fatty acid profile were analyzed on a significance level of 0.05 with oneway ANOVA and post hoc analysis that comprised pairwise multiple comparisons conducted with the Tukey's honestly significant difference test. These calculations were performed with the SPSS (IBM SPSS Statistics, version 19.0, Chicago, IL, USA) statistical software for Windows.

2.7.2. Multivariate Data Analysis. The obtained results were divided into two datasets: one comprising NMR data and the other containing data from GC-FID. Each dataset was imported into the SIMCA-P version 13.0 (Umetrics, Umeå, Sweden) for multivariate statistical analysis. GC data were scaled to unit variance (UV scaling), while NMR data were Pareto scaled, in order to increase the impact of low intensity, but significant, resonant peaks on the model [20]. All statistical models were extracted at a confidence level of $95 \%$ using the means of calculated values.

\section{Results and Discussion}

3.1. Proximate and Fatty Acid Composition. Walnuts moisture, ash, protein, and fat contents were found as $3.95 \pm$ $0.24,2.02 \pm 0.32,15.23 \pm 1.26$, and $62.21 \pm 1.18 \%$ (wet basis), respectively. The above results were higher to those reported by $\mathrm{Ma}$ et al. [21] for total lipid and lower for crude protein content. Irradiation caused insignificant $(P>0.05)$ change of moisture, ash, protein and fat content of walnuts, irrespective to the packaging. This statement is in accordance with the findings of Gecgel et al. [7] and Al-Bachir [22] who reported that there were no significant differences in moisture, ash, protein, and fat contents among nonirradiated and irradiated walnuts. Since similar results have also been reported for other nuts after irradiation [23], it is evident that radiation treatment does not cause significant changes in the principal nuts' components due to low moisture content, which prevents the formation of free radicals and the degradation of bioactive compounds.

Gas chromatography (GC) analysis of fatty acid methyl esters from nonirradiated and irradiated walnuts lipids revealed the presence of 31 fatty acids (Tables 1-3). Fatty acid percentages decreased in the order of polyunsaturated $>$ monounsaturated $>$ saturated (PUFA $>$ MUFA $>$ SFA). The fatty acid profile mainly consisted of the PUFA linoleic

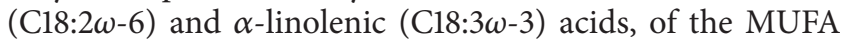
oleic acid (C18:1 $\omega-9)$ along with substantial proportions of the SFA palmitic (C16:0) and stearic (C18:0) acids.

The main results observed between nonirradiated and irradiated walnuts' total lipid fatty acids, irrespective of the packaging material, were as follows: (i) the proportion of saturated fatty acids (SFA) was gradually $(P<0.05)$ increased at 5, 10, and $13 \mathrm{kGy}$, along with a parallel decrease of polyunsaturated (PUFA) fatty acid; (ii) the PUFA/SFA and MUFA/SFA ratios were significantly decreased at all doses; and (iii) the C18:1 $\omega-9 / \mathrm{C} 18: 2 \omega-6$ ratio was not affected by irradiation treatment. These results were verified by similar studies on the effect of radiation treatment in nuts lipids by other researchers $[14,23,24]$. Polyunsaturated fatty acid proportion reduction may be due to their partial hydrogenation from hydrogen ions generated by irradiation, which causes a concomitant increase in SFA proportion.

The main results observed in relation to packaging conditions were as follows: (i) irradiation caused the highest $(P<0.05)$ SFA proportion increase in air packed irradiated walnuts TL and the lowest in MAP packed irradiated walnuts TL; (ii) MUFA proportion showed the highest decrease in vacuum packed irradiated walnuts TL; and (iii) irradiation evoked the highest $(P<0.05) \omega-6$ and $\omega-3$ fatty acids proportion decrease in air packed irradiated walnuts TL and the lowest in MAP packed irradiated walnuts TL.

Concerning fatty acid ratios, irradiation caused (i) the highest $(P<0.05)$ PUFA/SFA ratio decrease in air packed irradiated walnuts TL and the lowest in MAP packed irradiated walnuts TL, (ii) significant $(P<0.05)$ decrease of PUFA/MUFA ratio only in air and MAP packed irradiated walnuts TL, (iii) the highest $(P<0.05)$ MUFA/SFA ratio decrease in air and vacuum packed irradiated walnuts TL, and (iv) the highest $(P<0.05) \omega-6 / \omega-3$ fatty acids' ratio increase in air packed irradiated walnuts TL.

It is, therefore, concluded that the calculated fatty acid proportions are influenced by irradiation dose and packaging material, confirming different effect of packaging type on FA profile when they interact with radiation process.

Principal component analysis (PCA) was used to further explore the above results. A PCA model with two components was computed on the total set of nonirradiated and irradiated total lipids' samples in all packaging conditions (Figures 1(a), 1(b), and 1(c)). The first and second principal components 
TABLE 1: Fatty acids composition \% of (Total Fatty Acids) TFA in total lipids of non-irradiated and irradiated walnuts packaged in atmospheric air.

\begin{tabular}{|c|c|c|c|c|}
\hline Fatty acids & $0 \mathrm{kGy}$ & 5 kGy & $10 \mathrm{kGy}$ & 13 kGy \\
\hline $\mathrm{C} 12: 0$ & $0.03 \pm 0.00^{\mathrm{a}}$ & $0.03 \pm 0.00^{\mathrm{a}}$ & $0.03 \pm 0.00^{\mathrm{a}}$ & $0.03 \pm 0.00^{\mathrm{a}}$ \\
\hline C14:0 & $0.04 \pm 0.00^{\mathrm{a}}$ & $0.04 \pm 0.00^{\mathrm{a}}$ & $0.04 \pm 0.00^{\mathrm{a}}$ & $0.04 \pm 0.00^{\mathrm{a}}$ \\
\hline C14:1 & $0.02 \pm 0.00^{\mathrm{a}}$ & $0.02 \pm 0.00^{\mathrm{a}}$ & $0.02 \pm 0.00^{\mathrm{a}}$ & $0.02 \pm 0.00^{\mathrm{a}}$ \\
\hline iso-C15:0 & $0.01 \pm 0.00^{\mathrm{a}}$ & $0.01 \pm 0.00^{\mathrm{a}}$ & $0.01 \pm 0.00^{\mathrm{a}}$ & $0.01 \pm 0.00^{\mathrm{a}}$ \\
\hline $\mathrm{C} 15: 0$ & $0.35 \pm 0.01^{\mathrm{a}}$ & $0.34 \pm 0.01^{\mathrm{a}}$ & $0.35 \pm 0.01^{\mathrm{a}}$ & $0.39 \pm 0.01^{\mathrm{b}}$ \\
\hline $\mathrm{C} 15: 1 \omega-5$ & $0.01 \pm 0.00^{\mathrm{a}}$ & $0.01 \pm 0.00^{\mathrm{a}}$ & $0.01 \pm 0.00^{\mathrm{a}}$ & $0.01 \pm 0.00^{\mathrm{a}}$ \\
\hline $\mathrm{C} 16: 0$ & $6.76 \pm 0.07^{\mathrm{a}}$ & $7.45 \pm 0.06^{\mathrm{b}}$ & $8.22 \pm 0.04^{c}$ & $9.03 \pm 0.06^{\mathrm{d}}$ \\
\hline iso-C16:0 & $0.06 \pm 0.00^{\mathrm{a}}$ & $0.07 \pm 0.01^{\mathrm{a}}$ & $0.07 \pm 0.01^{\mathrm{a}}$ & $0.08 \pm 0.00^{\mathrm{b}}$ \\
\hline $\mathrm{C} 16: 1 \omega-7$ & $0.08 \pm 0.01^{\mathrm{a}}$ & $0.09 \pm 0.00^{\mathrm{a}}$ & $0.09 \pm 0.00^{\mathrm{a}}$ & $0.09 \pm 0.01^{\mathrm{a}}$ \\
\hline iso-C17:0 & - & $0.01 \pm 0.00^{\mathrm{a}}$ & $0.01 \pm 0.00^{\mathrm{a}}$ & $0.01 \pm 0.00^{\mathrm{a}}$ \\
\hline anteiso-C17:0 & $0.01 \pm 0.00^{\mathrm{a}}$ & $0.02 \pm 0.00^{\mathrm{b}}$ & $0.01 \pm 0.00^{\mathrm{a}}$ & $0.02 \pm 0.00^{\mathrm{b}}$ \\
\hline cyclo-C17:0 & $0.01 \pm 0.00^{\mathrm{a}}$ & $0.01 \pm 0.00^{\mathrm{a}}$ & $0.01 \pm 0.00^{\mathrm{a}}$ & $0.01 \pm 0.00^{\mathrm{a}}$ \\
\hline $\mathrm{C} 17: 0$ & $0.03 \pm 0.00^{\mathrm{a}}$ & $0.05 \pm 0.00^{b}$ & $0.03 \pm 0.00^{\mathrm{a}}$ & $0.06 \pm 0.01^{b}$ \\
\hline$C 17: 1 \omega-7$ & $0.02 \pm 0.00^{\mathrm{a}}$ & $0.02 \pm 0.00^{\mathrm{a}}$ & $0.02 \pm 0.00^{\mathrm{a}}$ & $0.02 \pm 0.00^{\mathrm{a}}$ \\
\hline C18:0 & $2.30 \pm 0.03^{\mathrm{a}}$ & $3.07 \pm 0.02^{\mathrm{b}}$ & $3.44 \pm 0.08^{\mathrm{c}}$ & $3.84 \pm 0.06^{\mathrm{d}}$ \\
\hline$C 18: 1 \omega-9$ & $19.77 \pm 0.07^{\mathrm{a}}$ & $19.36 \pm 0.07^{b}$ & $19.15 \pm 0.06^{c}$ & $19.21 \pm 0.05^{c}$ \\
\hline$C 18: 1 \omega-7$ & $0.52 \pm 0.01^{\mathrm{ab}}$ & $0.51 \pm 0.02^{\mathrm{ab}}$ & $0.49 \pm 0.01^{\mathrm{a}}$ & $0.52 \pm 0.01^{\mathrm{b}}$ \\
\hline $\mathrm{C} 18: 2 \omega-6$ & $59.71 \pm 0.16^{\mathrm{a}}$ & $59.01 \pm 0.06^{\mathrm{b}}$ & $58.44 \pm 0.06^{\mathrm{c}}$ & $57.45 \pm 0.04^{\mathrm{d}}$ \\
\hline $\mathrm{C} 18: 3 \omega-6$ & $0.07 \pm 0.00^{\mathrm{a}}$ & $0.07 \pm 0.01^{\mathrm{a}}$ & $0.05 \pm 0.01^{\mathrm{b}}$ & $0.03 \pm 0.00^{c}$ \\
\hline $\mathrm{C} 18: 3 \omega-3$ & $9.52 \pm 0.02^{\mathrm{a}}$ & $9.18 \pm 0.04^{b}$ & $8.90 \pm 0.08^{c}$ & $8.57 \pm 0.06^{\mathrm{d}}$ \\
\hline$C 18: 4 \omega-3$ & $0.06 \pm 0.00^{\mathrm{a}}$ & $0.06 \pm 0.00^{\mathrm{a}}$ & $0.04 \pm 0.00^{\mathrm{b}}$ & $0.03 \pm 0.00^{c}$ \\
\hline C19:0 & $0.01 \pm 0.00^{\mathrm{a}}$ & $0.01 \pm 0.00^{\mathrm{a}}$ & $0.01 \pm 0.00^{\mathrm{a}}$ & $0.01 \pm 0.00^{\mathrm{a}}$ \\
\hline C20:0 & $0.01 \pm 0.00^{\mathrm{a}}$ & $0.01 \pm 0.00^{\mathrm{a}}$ & $0.01 \pm 0.00^{\mathrm{a}}$ & $0.02 \pm 0.00^{\mathrm{b}}$ \\
\hline$C 20: 1 \omega-9$ & $0.17 \pm 0.00^{\mathrm{a}}$ & $0.16 \pm 0.01^{\mathrm{a}}$ & $0.18 \pm 0.01^{\mathrm{ab}}$ & $0.19 \pm 0.01^{b}$ \\
\hline $\mathrm{C} 20: 3 \omega-6$ & $0.02 \pm 0.00^{\mathrm{a}}$ & $0.02 \pm 0.00^{\mathrm{a}}$ & $0.02 \pm 0.00^{\mathrm{a}}$ & $0.01 \pm 0.01^{\mathrm{a}}$ \\
\hline $\mathrm{C} 20: 4 \omega-6$ & $0.01 \pm 0.00^{\mathrm{a}}$ & $0.01 \pm 0.00^{\mathrm{a}}$ & $0.01 \pm 0.00^{\mathrm{a}}$ & $0.01 \pm 0.00^{\mathrm{a}}$ \\
\hline $\mathrm{C} 20: 5 \omega-3$ & $0.02 \pm 0.00^{\mathrm{a}}$ & $0.02 \pm 0.00^{\mathrm{a}}$ & $0.01 \pm 0.00^{\mathrm{b}}$ & $0.01 \pm 0.00^{\mathrm{b}}$ \\
\hline $\mathrm{C} 22: 4 \omega-6$ & $0.02 \pm 0.00^{\mathrm{a}}$ & $0.02 \pm 0.00^{\mathrm{a}}$ & $0.01 \pm 0.00^{\mathrm{b}}$ & - \\
\hline $\mathrm{C} 22: 5 \omega-6$ & $0.03 \pm 0.00^{\mathrm{a}}$ & $0.02 \pm 0.00^{\mathrm{b}}$ & $0.01 \pm 0.00^{c}$ & $0.01 \pm 0.00^{\mathrm{c}}$ \\
\hline $\mathrm{C} 22: 5 \omega-3$ & $0.01 \pm 0.00^{\mathrm{a}}$ & $0.01 \pm 0.00^{\mathrm{a}}$ & $0.01 \pm 0.00^{\mathrm{a}}$ & - \\
\hline $\mathrm{C} 22: 6 \omega-3$ & $0.32 \pm 0.01^{\mathrm{a}}$ & $0.31 \pm 0.01^{\mathrm{a}}$ & $0.30 \pm 0.01^{\mathrm{a}}$ & $0.27 \pm 0.01^{\mathrm{b}}$ \\
\hline SFA & $9.62 \pm 0.10^{\mathrm{a}}$ & $11.12 \pm 0.06^{\mathrm{b}}$ & $12.22 \pm 0.11^{\mathrm{c}}$ & $13.56 \pm 0.06^{\mathrm{d}}$ \\
\hline MUFA & $20.59 \pm 0.05^{\mathrm{a}}$ & $20.16 \pm 0.08^{\mathrm{b}}$ & $19.96 \pm 0.07^{c}$ & $20.06 \pm 0.04^{\mathrm{bc}}$ \\
\hline PUFA & $69.79 \pm 0.15^{\mathrm{a}}$ & $68.72 \pm 0.03^{b}$ & $67.80 \pm 0.05^{c}$ & $66.38 \pm 0.04^{\mathrm{d}}$ \\
\hline UFA & $90.38 \pm 0.10^{\mathrm{a}}$ & $88.88 \pm 0.07^{\mathrm{b}}$ & $87.77 \pm 0.12^{c}$ & $86.44 \pm 0.06^{\mathrm{d}}$ \\
\hline$\Sigma \omega-6$ & $59.86 \pm 0.17^{\mathrm{a}}$ & $59.14 \pm 0.04^{\mathrm{b}}$ & $58.55 \pm 0.06^{\mathrm{c}}$ & $57.50 \pm 0.04^{\mathrm{d}}$ \\
\hline$\sum \omega-3$ & $9.93 \pm 0.02^{\mathrm{a}}$ & $9.57 \pm 0.04^{b}$ & $9.26 \pm 0.08^{c}$ & $8.88 \pm 0.06^{\mathrm{d}}$ \\
\hline$\omega-6 / \omega-3$ & $6.03 \pm 0.03^{\mathrm{a}}$ & $6.18 \pm 0.03^{\mathrm{b}}$ & $6.33 \pm 0.06^{\mathrm{c}}$ & $6.47 \pm 0.05^{\mathrm{d}}$ \\
\hline MUFA/SFA & $2.14 \pm 0.02^{\mathrm{a}}$ & $1.81 \pm 0.02^{\mathrm{b}}$ & $1.63 \pm 0.02^{c}$ & $1.48 \pm 0.01^{\mathrm{d}}$ \\
\hline PUFA/MUFA & $3.39 \pm 0.02^{\mathrm{a}}$ & $3.41 \pm 0.02^{\mathrm{a}}$ & $3.40 \pm 0.01^{\mathrm{a}}$ & $3.31 \pm 0.01^{\mathrm{b}}$ \\
\hline PUFA/SFA & $7.26 \pm 0.09^{\mathrm{a}}$ & $6.18 \pm 0.04^{b}$ & $5.55 \pm 0.05^{c}$ & $4.90 \pm 0.02^{\mathrm{d}}$ \\
\hline $\mathrm{C} 18: 1 \omega-9 / \mathrm{C} 18: 2 \omega-6$ & $0.33 \pm 0.00^{\mathrm{a}}$ & $0.33 \pm 0.00^{\mathrm{a}}$ & $0.33 \pm 0.00^{\mathrm{a}}$ & $0.33 \pm 0.00^{\mathrm{a}}$ \\
\hline $\mathrm{C} 16: 0 / \mathrm{C} 18: 0$ & $2.94 \pm 0.01^{\mathrm{a}}$ & $2.43 \pm 0.01^{\mathrm{b}}$ & $2.39 \pm 0.04^{\mathrm{cb}}$ & $2.35 \pm 0.05^{\mathrm{c}}$ \\
\hline
\end{tabular}

Values are the mean of three determinations from two individual experiments $(n=6)$.

Means in the same row bearing different letters differ significantly $(P<0.05)$.

(PC1 and PC2) accounted for $59.4 \%$ and $12 \%$ of the variation, respectively. Nonirradiated samples were discriminated from the rest of the samples along the second principal component and were located in the 2 nd quadrant, mainly characterized

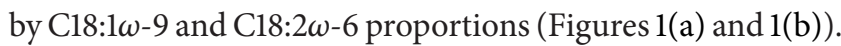
According to the contribution plot (Figure 1(c)) it seems that irradiated walnuts with 5,10 , and $13 \mathrm{kGy}$ doses were primarily influenced by $\mathrm{C} 16: 1 \omega-7$ and iso-C17:0, and secondarily by 
TABLE 2: Fatty acids composition \% of (Total Fatty Acids) TFA in total lipids of non-irradiated and irradiated walnuts packaged in vacuum.

\begin{tabular}{|c|c|c|c|c|}
\hline Fatty acids & 0 kGy & $5 \mathrm{kGy}$ & 10 kGy & 13 kGy \\
\hline $\mathrm{C} 12: 0$ & $0.03 \pm 0.00^{\mathrm{a}}$ & $0.03 \pm 0.00^{\mathrm{a}}$ & $0.03 \pm 0.00^{\mathrm{a}}$ & $0.03 \pm 0.00^{\mathrm{a}}$ \\
\hline C14:0 & $0.04 \pm 0.00^{\mathrm{a}}$ & $0.04 \pm 0.00^{\mathrm{a}}$ & $0.04 \pm 0.00^{\mathrm{a}}$ & $0.04 \pm 0.00^{\mathrm{a}}$ \\
\hline C14:1 & $0.02 \pm 0.00^{\mathrm{a}}$ & $0.02 \pm 0.00^{\mathrm{a}}$ & $0.02 \pm 0.00^{\mathrm{a}}$ & $0.02 \pm 0.00^{\mathrm{a}}$ \\
\hline iso- $\mathrm{C} 15: 0$ & $0.01 \pm 0.00^{\mathrm{a}}$ & $0.01 \pm 0.00^{\mathrm{a}}$ & $0.01 \pm 0.00^{\mathrm{a}}$ & $0.01 \pm 0.00^{\mathrm{a}}$ \\
\hline $\mathrm{C} 15: 0$ & $0.35 \pm 0.01^{\mathrm{a}}$ & $0.35 \pm 0.01^{\mathrm{a}}$ & $0.35 \pm 0.01^{\mathrm{a}}$ & $0.39 \pm 0.01^{b}$ \\
\hline $\mathrm{C} 15: 1 \omega-5$ & $0.01 \pm 0.00^{\mathrm{a}}$ & $0.01 \pm 0.00^{\mathrm{a}}$ & $0.01 \pm 0.00^{\mathrm{a}}$ & $0.01 \pm 0.00^{\mathrm{a}}$ \\
\hline $\mathrm{C} 16: 0$ & $6.76 \pm 0.07^{\mathrm{a}}$ & $7.25 \pm 0.04^{\mathrm{b}}$ & $8.20 \pm 0.04^{c}$ & $9.41 \pm 0.02^{\mathrm{d}}$ \\
\hline iso-C16:0 & $0.06 \pm 0.00^{\mathrm{a}}$ & $0.07 \pm 0.01^{\mathrm{a}}$ & $0.07 \pm 0.01^{\mathrm{a}}$ & $0.10 \pm 0.01^{b}$ \\
\hline$C 16: 1 \omega-7$ & $0.08 \pm 0.01^{\mathrm{a}}$ & $0.09 \pm 0.00^{\mathrm{a}}$ & $0.09 \pm 0.00^{\mathrm{a}}$ & $0.09 \pm 0.01^{\mathrm{a}}$ \\
\hline iso- $\mathrm{C} 17: 0$ & - & $0.01 \pm 0.00^{\mathrm{a}}$ & $0.01 \pm 0.00^{\mathrm{a}}$ & $0.01 \pm 0.00^{\mathrm{a}}$ \\
\hline anteiso-C17:0 & $0.01 \pm 0.00^{\mathrm{a}}$ & $0.02 \pm 0.00^{\mathrm{b}}$ & $0.01 \pm 0.00^{\mathrm{a}}$ & $0.02 \pm 0.00^{\mathrm{b}}$ \\
\hline cyclo-C17:0 & $0.01 \pm 0.00^{\mathrm{a}}$ & $0.01 \pm 0.00^{\mathrm{a}}$ & $0.01 \pm 0.00^{\mathrm{a}}$ & $0.01 \pm 0.00^{\mathrm{a}}$ \\
\hline $\mathrm{C} 17: 0$ & $0.03 \pm 0.00^{\mathrm{a}}$ & $0.05 \pm 0.00^{\mathrm{b}}$ & $0.05 \pm 0.00^{\mathrm{b}}$ & $0.06 \pm 0.01^{b}$ \\
\hline$C 17: 1 \omega-7$ & $0.02 \pm 0.00^{\mathrm{a}}$ & $0.02 \pm 0.00^{\mathrm{a}}$ & $0.02 \pm 0.00^{\mathrm{a}}$ & $0.02 \pm 0.00^{\mathrm{a}}$ \\
\hline C18:0 & $2.30 \pm 0.03^{\mathrm{a}}$ & $2.57 \pm 0.04^{\mathrm{b}}$ & $2.75 \pm 0.08^{c}$ & $3.14 \pm 0.07^{\mathrm{d}}$ \\
\hline$C 18: 1 \omega-9$ & $19.77 \pm 0.07^{\mathrm{a}}$ & $19.62 \pm 0.04^{\mathrm{b}}$ & $19.30 \pm 0.04^{\mathrm{c}}$ & $18.93 \pm 0.03^{\mathrm{d}}$ \\
\hline$C 18: 1 \omega-7$ & $0.52 \pm 0.01^{\mathrm{a}}$ & $0.53 \pm 0.02^{\mathrm{a}}$ & $0.50 \pm 0.01^{\mathrm{ab}}$ & $0.47 \pm 0.02^{\mathrm{b}}$ \\
\hline $\mathrm{C} 18: 2 \omega-6$ & $59.71 \pm 0.16^{\mathrm{a}}$ & $59.19 \pm 0.05^{\mathrm{b}}$ & $58.75 \pm 0.10^{c}$ & $57.64 \pm 0.05^{\mathrm{d}}$ \\
\hline $\mathrm{C} 18: 3 \omega-6$ & $0.07 \pm 0.00^{\mathrm{a}}$ & $0.07 \pm 0.01^{\mathrm{a}}$ & $0.05 \pm 0.00^{\mathrm{b}}$ & $0.05 \pm 0.00^{\mathrm{b}}$ \\
\hline $\mathrm{C} 18: 3 \omega-3$ & $9.52 \pm 0.02^{\mathrm{a}}$ & $9.36 \pm 0.05^{\mathrm{b}}$ & $9.08 \pm 0.06^{c}$ & $8.90 \pm 0.06^{\mathrm{d}}$ \\
\hline $\mathrm{C} 18: 4 \omega-3$ & $0.06 \pm 0.00^{\mathrm{a}}$ & $0.06 \pm 0.00^{\mathrm{a}}$ & $0.05 \pm 0.00^{\mathrm{b}}$ & $0.06 \pm 0.00^{\mathrm{a}}$ \\
\hline C19:0 & $0.01 \pm 0.00^{\mathrm{a}}$ & $0.01 \pm 0.00^{\mathrm{a}}$ & $0.01 \pm 0.00^{\mathrm{a}}$ & $0.01 \pm 0.00^{\mathrm{a}}$ \\
\hline C20:0 & $0.01 \pm 0.00^{\mathrm{a}}$ & $0.01 \pm 0.00^{\mathrm{a}}$ & $0.01 \pm 0.00^{\mathrm{a}}$ & $0.02 \pm 0.01^{\mathrm{a}}$ \\
\hline C20:1 $\omega-9$ & $0.17 \pm 0.00^{\mathrm{a}}$ & $0.17 \pm 0.01^{\mathrm{a}}$ & $0.17 \pm 0.01^{\mathrm{a}}$ & $0.19 \pm 0.00^{\mathrm{b}}$ \\
\hline $\mathrm{C} 20: 3 \omega-6$ & $0.02 \pm 0.00^{\mathrm{a}}$ & $0.02 \pm 0.00^{\mathrm{a}}$ & $0.02 \pm 0.00^{\mathrm{a}}$ & $0.02 \pm 0.00^{\mathrm{a}}$ \\
\hline $\mathrm{C} 20: 4 \omega-6$ & $0.01 \pm 0.00^{\mathrm{a}}$ & $0.01 \pm 0.00^{\mathrm{a}}$ & $0.01 \pm 0.00^{\mathrm{a}}$ & $0.01 \pm 0.00^{\mathrm{a}}$ \\
\hline $\mathrm{C} 20: 5 \omega-3$ & $0.02 \pm 0.00^{\mathrm{a}}$ & $0.02 \pm 0.00^{\mathrm{a}}$ & $0.01 \pm 0.00^{\mathrm{b}}$ & $0.01 \pm 0.00^{\mathrm{b}}$ \\
\hline $\mathrm{C} 22: 4 \omega-6$ & $0.02 \pm 0.00^{\mathrm{a}}$ & $0.02 \pm 0.00^{\mathrm{a}}$ & $0.02 \pm 0.00^{\mathrm{a}}$ & $0.01 \pm 0.00^{\mathrm{b}}$ \\
\hline $\mathrm{C} 22: 5 \omega-6$ & $0.03 \pm 0.00^{\mathrm{a}}$ & $0.03 \pm 0.00^{\mathrm{a}}$ & $0.02 \pm 0.00^{\mathrm{b}}$ & $0.02 \pm 0.00^{\mathrm{b}}$ \\
\hline $\mathrm{C} 22: 5 \omega-3$ & $0.01 \pm 0.00^{\mathrm{a}}$ & $0.01 \pm 0.00^{\mathrm{a}}$ & $0.01 \pm 0.00^{\mathrm{a}}$ & $0.01 \pm 0.00^{\mathrm{a}}$ \\
\hline $\mathrm{C} 22: 6 \omega-3$ & $0.32 \pm 0.01^{\mathrm{a}}$ & $0.32 \pm 0.01^{\mathrm{a}}$ & $0.31 \pm 0.01^{\mathrm{ab}}$ & $0.29 \pm 0.01^{b}$ \\
\hline SFA & $9.62 \pm 0.10^{\mathrm{a}}$ & $10.44 \pm 0.02^{\mathrm{b}}$ & $11.55 \pm 0.06^{\mathrm{c}}$ & $13.25 \pm 0.05^{\mathrm{d}}$ \\
\hline MUFA & $20.59 \pm 0.05^{\mathrm{a}}$ & $20.46 \pm 0.05^{\mathrm{b}}$ & $20.11 \pm 0.03^{c}$ & $19.73 \pm 0.04^{\mathrm{d}}$ \\
\hline PUFA & $69.79 \pm 0.15^{\mathrm{a}}$ & $69.10 \pm 0.07^{\mathrm{b}}$ & $68.34 \pm 0.05^{\mathrm{c}}$ & $67.02 \pm 0.05^{\mathrm{d}}$ \\
\hline UFA & $90.38 \pm 0.10^{\mathrm{a}}$ & $89.56 \pm 0.04^{\mathrm{b}}$ & $88.45 \pm 0.06^{c}$ & $86.75 \pm 0.05^{\mathrm{d}}$ \\
\hline$\sum \omega-6$ & $59.86 \pm 0.17^{\mathrm{a}}$ & $59.33 \pm 0.04^{\mathrm{b}}$ & $58.87 \pm 0.10^{\mathrm{C}}$ & $57.75 \pm 0.05^{\mathrm{d}}$ \\
\hline$\sum \omega-3$ & $9.93 \pm 0.02^{\mathrm{a}}$ & $9.77 \pm 0.04^{\mathrm{b}}$ & $9.46 \pm 0.05^{c}$ & $9.27 \pm 0.05^{\mathrm{d}}$ \\
\hline$\omega-6 / \omega-3$ & $6.03 \pm 0.03^{\mathrm{a}}$ & $6.07 \pm 0.02^{\mathrm{a}}$ & $6.22 \pm 0.04^{\mathrm{b}}$ & $6.23 \pm 0.03^{b}$ \\
\hline MUFA/SFA & $2.14 \pm 0.02^{\mathrm{a}}$ & $1.96 \pm 0.01^{b}$ & $1.74 \pm 0.02^{\mathrm{c}}$ & $1.49 \pm 0.01^{\mathrm{d}}$ \\
\hline PUFA/MUFA & $3.39 \pm 0.02^{\mathrm{a}}$ & $3.38 \pm 0.01^{\mathrm{a}}$ & $3.40 \pm 0.01^{\mathrm{a}}$ & $3.40 \pm 0.01^{\mathrm{a}}$ \\
\hline PUFA/SFA & $7.26 \pm 0.09^{\mathrm{a}}$ & $6.62 \pm 0.02^{b}$ & $5.92 \pm 0.04^{\mathrm{c}}$ & $5.06 \pm 0.02^{\mathrm{d}}$ \\
\hline $\mathrm{C} 18: 1 \omega-9 / \mathrm{C} 18: 2 \omega-6$ & $0.33 \pm 0.00^{\mathrm{a}}$ & $0.33 \pm 0.00^{\mathrm{a}}$ & $0.33 \pm 0.00^{\mathrm{a}}$ & $0.33 \pm 0.00^{\mathrm{a}}$ \\
\hline $\mathrm{C} 16: 0 / \mathrm{C} 18: 0$ & $2.94 \pm 0.01^{\mathrm{a}}$ & $2.82 \pm 0.06^{\mathrm{b}}$ & $2.98 \pm 0.09^{\mathrm{a}}$ & $3.00 \pm 0.07^{\mathrm{a}}$ \\
\hline
\end{tabular}

Values are the mean of three determinations from two individual experiments $(n=6)$.

Means in the same row bearing different letters differ significantly $(P<0.05)$.

iso-C16:0, $\mathrm{C} 17: 0$, and anteiso-C17:0, which tend to increase with the irradiation dose. Apart from the control group, the clearest grouping was observed for samples irradiated at the dose of $5 \mathrm{kGy}$ situated in the 3rd quadrant, irrespective of the packaging conditions. These samples were mainly influenced

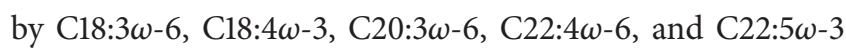
proportions. Samples irradiated at doses of 10 and $13 \mathrm{kGy}$ were discriminated from the rest of the samples and were located in the 1st and the 4th quadrants, except for irradiated samples at $10 \mathrm{kGy}$ in MAP package, which were located 
TABLE 3: Fatty acids composition \% of (Total Fatty Acids) TFA in total lipids of non-irradiated and irradiated walnuts packaged in modified atmosphere.

\begin{tabular}{|c|c|c|c|c|}
\hline Fatty acids & $0 \mathrm{kGy}$ & 5 kGy & $10 \mathrm{kGy}$ & $13 \mathrm{kGy}$ \\
\hline $\mathrm{C} 12: 0$ & $0.03 \pm 0.00^{\mathrm{a}}$ & $0.03 \pm 0.00^{\mathrm{a}}$ & $0.03 \pm 0.00^{\mathrm{a}}$ & $0.03 \pm 0.00^{\mathrm{a}}$ \\
\hline $\mathrm{C} 14: 0$ & $0.04 \pm 0.00^{\mathrm{a}}$ & $0.04 \pm 0.00^{\mathrm{a}}$ & $0.04 \pm 0.00^{\mathrm{a}}$ & $0.04 \pm 0.00^{\mathrm{a}}$ \\
\hline $\mathrm{C} 14: 1$ & $0.02 \pm 0.00^{\mathrm{a}}$ & $0.02 \pm 0.00^{\mathrm{a}}$ & $0.02 \pm 0.00^{\mathrm{a}}$ & $0.02 \pm 0.00^{\mathrm{a}}$ \\
\hline iso-C15:0 & $0.01 \pm 0.00^{\mathrm{a}}$ & $0.01 \pm 0.00^{\mathrm{a}}$ & $0.01 \pm 0.00^{\mathrm{a}}$ & $0.01 \pm 0.00^{\mathrm{a}}$ \\
\hline $\mathrm{C} 15: 0$ & $0.35 \pm 0.01^{\mathrm{a}}$ & $0.34 \pm 0.01^{\mathrm{a}}$ & $0.35 \pm 0.01^{\mathrm{a}}$ & $0.39 \pm 0.01^{\mathrm{b}}$ \\
\hline $\mathrm{C} 15: 1 \omega-5$ & $0.01 \pm 0.00^{\mathrm{a}}$ & $0.01 \pm 0.00^{\mathrm{a}}$ & $0.01 \pm 0.00^{\mathrm{a}}$ & $0.01 \pm 0.00^{\mathrm{a}}$ \\
\hline C16:0 & $6.76 \pm 0.07^{\mathrm{a}}$ & $7.02 \pm 0.12^{\mathrm{b}}$ & $7.22 \pm 0.06^{\mathrm{b}}$ & $7.45 \pm 0.07^{\mathrm{c}}$ \\
\hline iso-C16:0 & $0.06 \pm 0.00^{\mathrm{a}}$ & $0.07 \pm 0.01^{\mathrm{a}}$ & $0.07 \pm 0.01^{\mathrm{a}}$ & $0.08 \pm 0.00^{\mathrm{b}}$ \\
\hline $\mathrm{C} 16: 1 \omega-7$ & $0.08 \pm 0.01^{\mathrm{a}}$ & $0.09 \pm 0.00^{\mathrm{a}}$ & $0.09 \pm 0.00^{\mathrm{a}}$ & $0.09 \pm 0.01^{\mathrm{a}}$ \\
\hline iso-C17:0 & - & $0.01 \pm 0.00^{\mathrm{a}}$ & $0.01 \pm 0.00^{\mathrm{a}}$ & $0.01 \pm 0.00^{\mathrm{a}}$ \\
\hline anteiso-C17:0 & $0.01 \pm 0.00^{\mathrm{a}}$ & $0.02 \pm 0.00^{\mathrm{b}}$ & $0.01 \pm 0.00^{\mathrm{a}}$ & $0.02 \pm 0.00^{\mathrm{b}}$ \\
\hline cyclo-C17:0 & $0.01 \pm 0.00^{\mathrm{a}}$ & $0.01 \pm 0.00^{\mathrm{a}}$ & $0.01 \pm 0.00^{\mathrm{a}}$ & $0.01 \pm 0.00^{\mathrm{a}}$ \\
\hline $\mathrm{C} 17: 0$ & $0.03 \pm 0.00^{\mathrm{a}}$ & $0.05 \pm 0.00^{\mathrm{b}}$ & $0.03 \pm 0.00^{\mathrm{a}}$ & $0.04 \pm 0.01^{\mathrm{a}}$ \\
\hline $\mathrm{C} 17: 1 \omega-7$ & $0.02 \pm 0.00^{\mathrm{a}}$ & $0.02 \pm 0.00^{\mathrm{a}}$ & $0.02 \pm 0.00^{\mathrm{a}}$ & $0.02 \pm 0.00^{\mathrm{a}}$ \\
\hline C18:0 & $2.30 \pm 0.03^{\mathrm{a}}$ & $2.56 \pm 0.03^{\mathrm{b}}$ & $2.98 \pm 0.03^{c}$ & $3.54 \pm 0.03^{\mathrm{d}}$ \\
\hline $\mathrm{C} 18: 1 \omega-9$ & $19.77 \pm 0.07^{\mathrm{a}}$ & $19.59 \pm 0.14^{\mathrm{ab}}$ & $19.53 \pm 0.03^{\mathrm{b}}$ & $19.76 \pm 0.06^{\mathrm{a}}$ \\
\hline $\mathrm{C} 18: 1 \omega-7$ & $0.52 \pm 0.01^{\mathrm{a}}$ & $0.53 \pm 0.01^{\mathrm{a}}$ & $0.51 \pm 0.01^{\mathrm{a}}$ & $0.58 \pm 0.00^{\mathrm{b}}$ \\
\hline $\mathrm{C} 18: 2 \omega-6$ & $59.71 \pm 0.16^{\mathrm{a}}$ & $59.04 \pm 0.11^{\mathrm{b}}$ & $58.77 \pm 0.05^{\mathrm{c}}$ & $58.10 \pm 0.05^{\mathrm{d}}$ \\
\hline $\mathrm{C} 18: 3 \omega-6$ & $0.07 \pm 0.00^{\mathrm{a}}$ & $0.08 \pm 0.01^{\mathrm{a}}$ & $0.06 \pm 0.00^{\mathrm{b}}$ & $0.07 \pm 0.00^{\mathrm{a}}$ \\
\hline $\mathrm{C} 18: 3 \omega-3$ & $9.52 \pm 0.02^{\mathrm{a}}$ & $9.80 \pm 0.09^{b}$ & $9.58 \pm 0.04^{\mathrm{a}}$ & $9.07 \pm 0.05^{\mathrm{c}}$ \\
\hline $\mathrm{C} 18: 4 \omega-3$ & $0.06 \pm 0.00^{\mathrm{a}}$ & $0.06 \pm 0.00^{\mathrm{a}}$ & $0.05 \pm 0.00^{\mathrm{b}}$ & $0.06 \pm 0.00^{\mathrm{a}}$ \\
\hline $\mathrm{C} 19: 0$ & $0.01 \pm 0.00^{\mathrm{a}}$ & $0.01 \pm 0.00^{\mathrm{a}}$ & $0.01 \pm 0.00^{\mathrm{a}}$ & $0.01 \pm 0.00^{\mathrm{a}}$ \\
\hline C20:0 & $0.01 \pm 0.00^{\mathrm{a}}$ & $0.01 \pm 0.00^{\mathrm{a}}$ & $0.01 \pm 0.00^{\mathrm{a}}$ & $0.01 \pm 0.00^{\mathrm{a}}$ \\
\hline$C 20: 1 \omega-9$ & $0.17 \pm 0.00^{\mathrm{a}}$ & $0.16 \pm 0.01^{\mathrm{a}}$ & $0.18 \pm 0.01^{\mathrm{ab}}$ & $0.19 \pm 0.01^{\mathrm{b}}$ \\
\hline $\mathrm{C} 20: 3 \omega-6$ & $0.02 \pm 0.00^{\mathrm{a}}$ & $0.02 \pm 0.00^{\mathrm{a}}$ & $0.02 \pm 0.00^{\mathrm{a}}$ & $0.02 \pm 0.01^{\mathrm{a}}$ \\
\hline $\mathrm{C} 20: 4 \omega-6$ & $0.01 \pm 0.00^{\mathrm{a}}$ & $0.01 \pm 0.00^{\mathrm{a}}$ & $0.01 \pm 0.00^{\mathrm{a}}$ & $0.01 \pm 0.00^{\mathrm{a}}$ \\
\hline $\mathrm{C} 20: 5 \omega-3$ & $0.02 \pm 0.00^{\mathrm{a}}$ & $0.02 \pm 0.00^{\mathrm{a}}$ & $0.01 \pm 0.00^{\mathrm{b}}$ & $0.01 \pm 0.00^{\mathrm{b}}$ \\
\hline $\mathrm{C} 22: 4 \omega-6$ & $0.02 \pm 0.00^{\mathrm{a}}$ & $0.02 \pm 0.00^{\mathrm{a}}$ & $0.02 \pm 0.00^{\mathrm{a}}$ & $0.01 \pm 0.00^{\mathrm{b}}$ \\
\hline $\mathrm{C} 22: 5 \omega-6$ & $0.03 \pm 0.00^{\mathrm{a}}$ & $0.02 \pm 0.00^{\mathrm{a}}$ & $0.02 \pm 0.00^{\mathrm{a}}$ & $0.02 \pm 0.00^{\mathrm{a}}$ \\
\hline $\mathrm{C} 22: 5 \omega-3$ & $0.01 \pm 0.00^{\mathrm{a}}$ & $0.01 \pm 0.00^{\mathrm{a}}$ & $0.01 \pm 0.00^{\mathrm{a}}$ & $0.01 \pm 0.00^{\mathrm{a}}$ \\
\hline $\mathrm{C} 22: 6 \omega-3$ & $0.32 \pm 0.01^{\mathrm{a}}$ & $0.31 \pm 0.01^{\mathrm{a}}$ & $0.32 \pm 0.01^{\mathrm{a}}$ & $0.30 \pm 0.01^{\mathrm{a}}$ \\
\hline SFA & $9.62 \pm 0.10^{\mathrm{a}}$ & $10.19 \pm 0.15^{\mathrm{b}}$ & $10.76 \pm 0.03^{c}$ & $11.65 \pm 0.08^{\mathrm{d}}$ \\
\hline MUFA & $20.59 \pm 0.05^{\mathrm{ac}}$ & $20.42 \pm 0.13^{\mathrm{ab}}$ & $20.36 \pm 0.03^{b}$ & $20.67 \pm 0.05^{\mathrm{c}}$ \\
\hline PUFA & $69.79 \pm 0.15^{\mathrm{a}}$ & $69.40 \pm 0.09^{\mathrm{b}}$ & $68.86 \pm 0.05^{\mathrm{c}}$ & $67.68 \pm 0.05^{\mathrm{d}}$ \\
\hline UFA & $90.38 \pm 0.10^{\mathrm{a}}$ & $89.81 \pm 0.15^{\mathrm{b}}$ & $89.22 \pm 0.03^{\mathrm{c}}$ & $88.35 \pm 0.08^{\mathrm{d}}$ \\
\hline$\Sigma \omega-6$ & $59.86 \pm 0.17^{\mathrm{a}}$ & $59.20 \pm 0.11^{\mathrm{b}}$ & $58.89 \pm 0.05^{\mathrm{c}}$ & $58.23 \pm 0.05^{\mathrm{d}}$ \\
\hline$\sum \omega-3$ & $9.93 \pm 0.02^{\mathrm{a}}$ & $10.20 \pm 0.09^{\mathrm{b}}$ & $9.97 \pm 0.05^{\mathrm{a}}$ & $9.45 \pm 0.02^{c}$ \\
\hline$\omega-6 / \omega-3$ & $6.03 \pm 0.03^{\mathrm{a}}$ & $5.80 \pm 0.06^{\mathrm{b}}$ & $5.91 \pm 0.03^{c}$ & $6.16 \pm 0.02^{\mathrm{d}}$ \\
\hline MUFA/SFA & $2.14 \pm 0.02^{\mathrm{a}}$ & $2.00 \pm 0.05^{\mathrm{b}}$ & $1.89 \pm 0.03^{c}$ & $1.77 \pm 0.02^{\mathrm{d}}$ \\
\hline PUFA/MUFA & $3.39 \pm 0.02^{\mathrm{a}}$ & $3.40 \pm 0.02^{\mathrm{a}}$ & $3.38 \pm 0.01^{\mathrm{a}}$ & $3.27 \pm 0.02^{\mathrm{b}}$ \\
\hline PUFA/SFA & $7.26 \pm 0.09^{a}$ & $6.81 \pm 0.10^{\mathrm{b}}$ & $6.39 \pm 0.02^{c}$ & $5.81 \pm 0.04^{\mathrm{d}}$ \\
\hline$C 18: 1 \omega-9 / C 18: 2 \omega-6$ & $0.33 \pm 0.00^{\mathrm{a}}$ & $0.33 \pm 0.00^{\mathrm{a}}$ & $0.33 \pm 0.00^{\mathrm{a}}$ & $0.34 \pm 0.01^{\mathrm{a}}$ \\
\hline $\mathrm{C} 16: 0 / \mathrm{C} 18: 0$ & $2.94 \pm 0.01^{\mathrm{a}}$ & $2.73 \pm 0.02^{\mathrm{b}}$ & $2.42 \pm 0.04^{c}$ & $2.11 \pm 0.03^{\mathrm{d}}$ \\
\hline
\end{tabular}

Values are the mean of three determinations from two individual experiments $(n=6)$.

Means in the same row bearing different letters differ significantly $(P<0.05)$.

almost at the center of the model, nearly in the 2nd quadrant. As shown in Figure 1(a) the packaging parameter seems to influence the samples as irradiation dose increases, clearly separating them in the PCA model. More specifically, air packaging follows a specific trend from the 3rd quadrant at $5 \mathrm{kGy}$ towards the outer part of the 1st quadrant at $13 \mathrm{kGy}$. Vacuum and MAP packaging at high irradiation doses tend to be differentiated from corresponding ones at low doses 


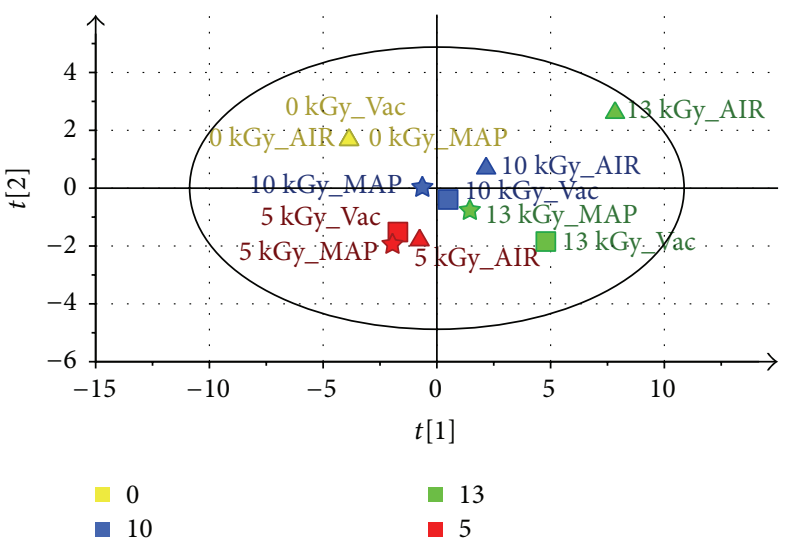

(a)

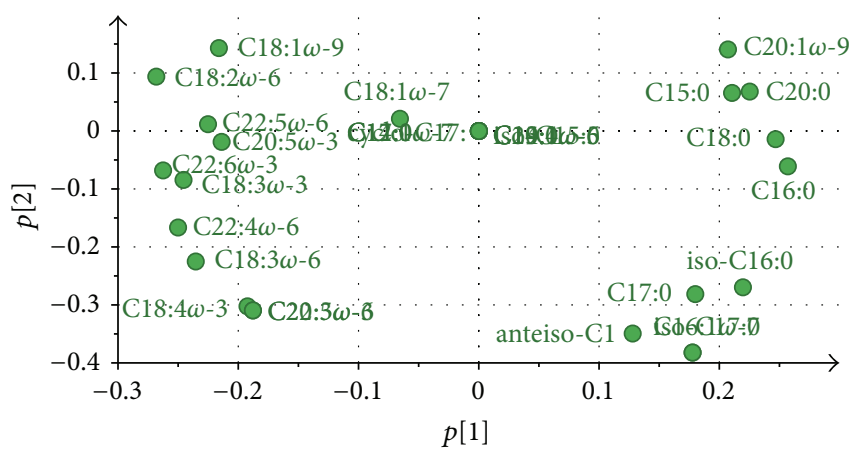

- $x$

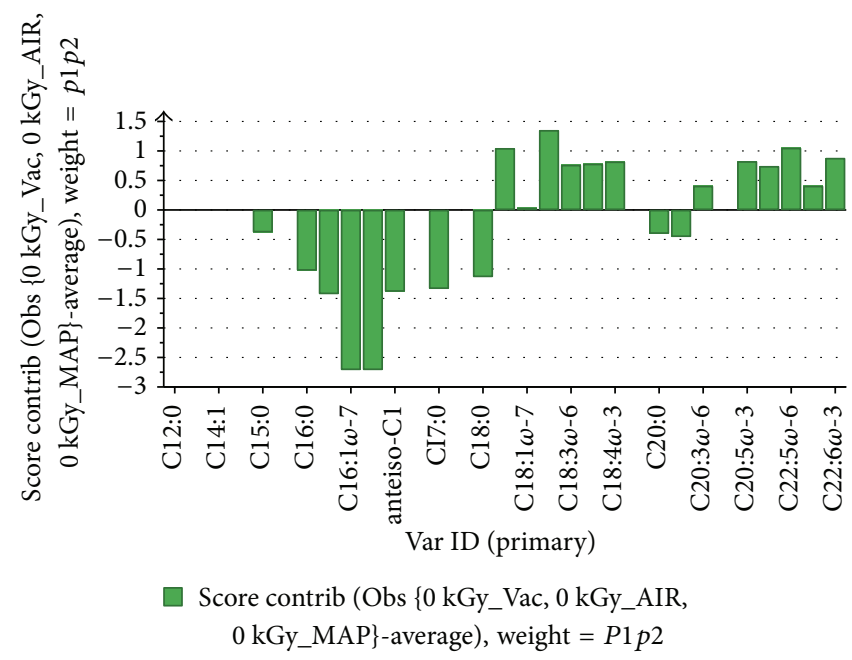

(c)

Figure 1: (a) PCA model of GC-FID data scaled to Unit Variance (UV scaling); $A=2, R^{2} X_{1,2 \text { (cum) }}: 71.5 \%$, and $Q^{2} \mathrm{X}_{1,2 \text { (cum) }}: 37.1 \%$. Samples of MAP, air, and vacuum packages are denoted by a star, triangle, and box, respectively. Yellow, red, blue, and green colors correspond to 0 , 5,10 , and $13 \mathrm{kGy}$, respectively, as also denoted by the labels. Only the mean values of six different measurements per irradiation dose and packaging are presented. (b) Loading plot. (c) Contribution plot indicating fatty acids responsible for the discrimination of nonirradiated control samples (positive values) with the irradiated ones (negative values).

but without following the previous trend. From the above results it is concluded that PCA analysis is a powerful tool to distinguish the samples according to the irradiation dose and the packaging conditions.

3.2. NMR Based Lipid Profile Analysis. The unsupervised PCA (principal components analysis) was applied on the Pareto scaled NMR data of the walnut samples in order to monitor possible trends in sample classification related to packaging type or irradiation dose. The PCA model (Figure 2(a)) extracted on the two first principal components (PCs) tends to discriminate between the irradiated samples $(5,10$, and $13 \mathrm{kGy})$ of MAP and air packages from the corresponding control samples ( $0 \mathrm{kGy})$ along PC1. Interestingly, the irradiation process seems to have minor impact on the vacuum samples. To a step further, a contribution plot was generated between the control samples and the irradiated ones (Figure 2(b)) so as to assess the impact of the irradiation process on the lipid profile of the samples. According to this plot, the nonirradiated samples are characterized by resonant peaks corresponding to the unsaturated fatty acids linolenic

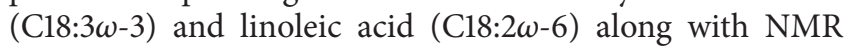
peaks belonging to methylene $-\mathrm{CH}_{2} \mathrm{CH}=\mathrm{CH}$ at $2.0 \mathrm{ppm}$ and olefinic- $\mathbf{C H}=\mathbf{C H}$-protons at $5.2 \mathrm{ppm}$. On the other hand, a resonant peak at $1.2 \mathrm{ppm}$, which is attributed to unsaturated fatty acids, characterized the irradiated sample as illustrated in the contribution plot. These outcomes are in accordance with those reported from GC-FID analysis demonstrating that increase of irradiation dose affects the quality of walnut lipids by decreasing PUFA and MUFA.

The discrimination of the packaging type was unveiled only after the exploration of the 3rd principal component (Figure 2(c)). The corresponding loading plot (Figure 2(d)) demonstrated that sn1,3 diglycerides (sn1,3 DG) along with some minor components (resonating at 5.4-6.5 ppm) characterize the samples located at the 1st and 2nd quadrants, 


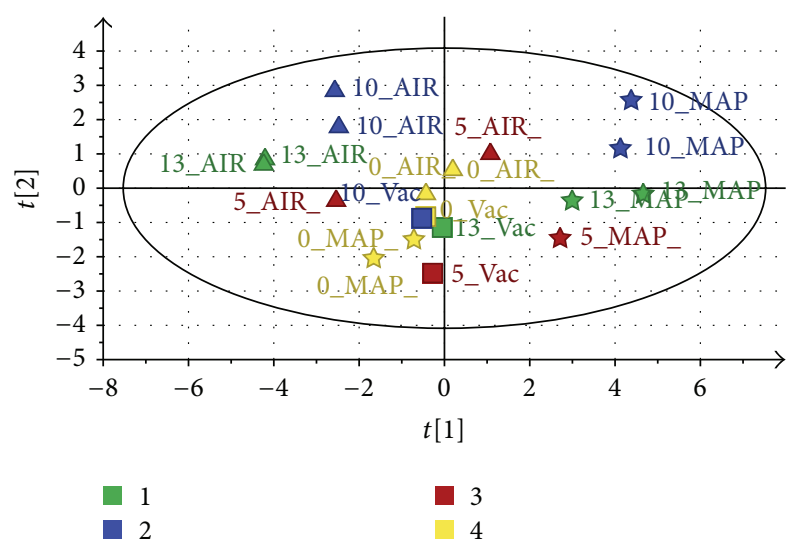

(a)

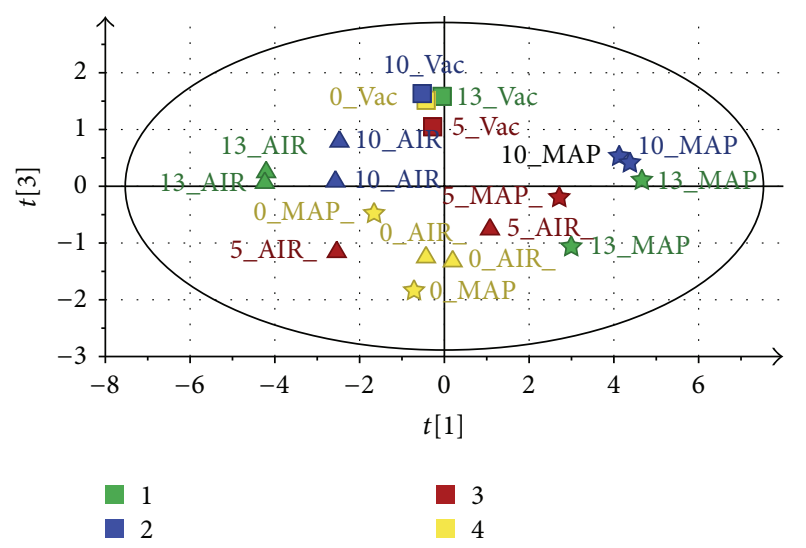

(c)

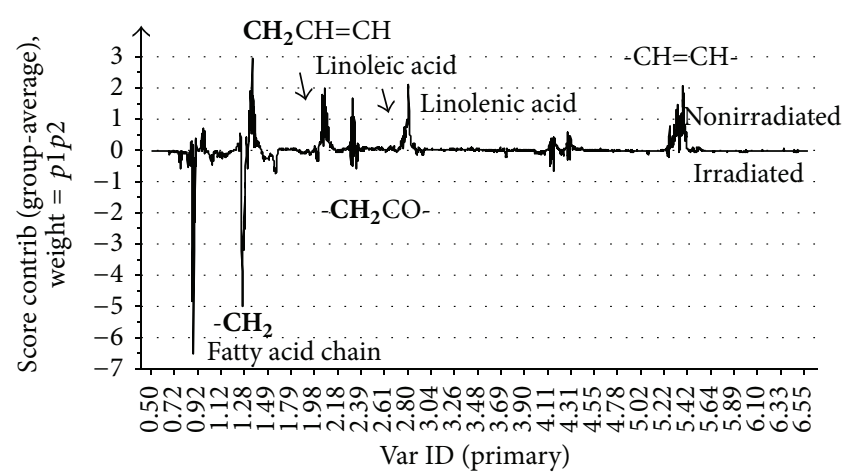

(b)

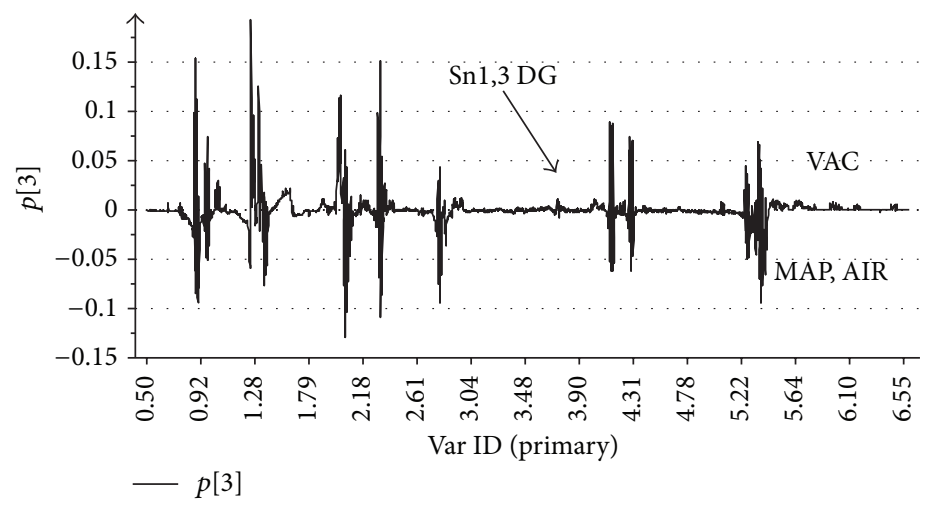

(d)

Figure 2: (a) PCA map of the two first PCs on NMR data using Pareto scaling of the total sample set. $A=3, R^{2} \mathrm{X}_{1,2}$ (cum): $77.5 \%$, and $Q^{2} \mathrm{X}_{1,2 \text { (cum) }}$ : $70.6 \%$. Samples of MAP, air, and vacuum packages are denoted by a star, triangle, and box, respectively. While yellow, red, blue, and green colors correspond to $0,5,10$, and $13 \mathrm{kGy}$, respectively, as also denoted by the labels. Only the mean values of each irradiation process are presented. (b) Contribution plot showing the difference between the average of the nonirradiated and irradiated samples. The variables with positive and negative values characterize the nonirradiated and the irradiated samples, respectively. (c) PCA map of PC1 and PC3 on NMR data of the total sample set. $R^{2} \mathrm{X}_{1,3 \text { (cum) }}: 61 \%$ and $Q^{2} \mathrm{X}_{1,3 \text { (cum) }}: 70.6 \%$. (d) Loading plot of PC3.

in our case vacuum samples of all irradiation doses and MAP and air samples of high irradiation doses (>10 kGy). This result may partially explain the grouping of the vacuum samples shown in Figure 2(a) indicating a deteriorated fatty acid profile. The presence of sn1,3 DG has been reported as a freshness/spoilage marker, shown to be abundant in nonfresh samples [25]. In our case the presence of sn1,3 DG is also related to irradiated samples at high doses.

\section{Conclusion}

This study reveals the effects of $\gamma$-irradiation on walnut's (Juglans regia L.) lipid fraction, using different doses and packaging conditions. GC-FID and NMR spectroscopy were implemented to evaluate fatty acid profiles and detect alterations capable of discriminating between irradiated samples. GC-FID results showed that SFA increased and MUFA and PUFA decreased with the increase of irradiation dose. Moreover, MUFA/SFA and PUFA/SFA ratios decreased $(P<$ 0.05) compared to control samples. It is concluded that the calculated fatty acid proportions are influenced by irradiation dose and packaging material, confirming different effect of packaging type on FA profile when interacting with radiation process. Unsupervised PCA on GC-FID and NMR data revealed that nonirradiated samples were discriminated from the rest of the samples and were mainly characterized by $\mathrm{C} 18: 1 \omega-9$ and $\mathrm{C} 18: 2 \omega-6$ proportions. Irradiated walnuts with 5,10 , and $13 \mathrm{kGy}$ doses were primarily influenced by C16:1 $\omega-7$ and iso-C17:0 and secondarily by iso-C16:0, $\mathrm{C} 17: 0$, and anteiso-C17:0, which tend to increase with the irradiation dose. The packaging parameter seems to influence the samples as irradiation dose increases. Particularly air packaging was the less protective means compared to MAP in respect to the fatty acid profiles. The presence of sn1,3 DG in irradiated samples of 10 and $13 \mathrm{kGy}$ also indicates deterioration of triglycerides. In conclusion, at irradiation doses of up to $5 \mathrm{kGy}$, the walnuts retained the nutritional benefits of its fatty acids, in particular MUFA and PUFA. Concerning the different types of packaging, greater stability in the nuts' lipid profile was observed using MAP packaging. 
From a nutritional point of view and in relation to the fatty acid alterations, MAP offered greater stability compared to vacuum and vacuum compared to air packaging.

\section{Abbreviations}

FAME: Fatty acid methyl esters

GC-FID: Gas chromatography-mass spectrometry

LDL: Low density lipoproteins

MAP: $\quad$ Modified atmosphere package

MUFA: Monounsaturated fatty acids

NMR: Nuclear magnetic resonance

PCA: Principal component analysis

PUFA: Polyunsaturated fatty acids

SFA: $\quad$ Saturated fatty acids

TL: Total lipids

UAE: Ultrasound assisted extraction.

\section{Conflict of Interests}

The authors declare that there is no conflict of interests regarding the publication of this paper.

\section{Acknowledgments}

This work is supported by the Programme ARHIMIDES 2012-2015, Sectoral Operational Programme, Education and Lifelong Learning (ESPA 2007-2013). The authors would like to acknowledge Dr. Panagiotis Zaverdinos from the Institute of Pharmaceutical Research and Technology (IPRT S.A.) (http://www.ifet.gr/english_site/) for providing the Cobalt 60 irradiation facilities.

\section{References}

[1] F. Shahidi and H. Miraliakbari, "Tree nut oils," in Bailey's Industrial Oil and Fat Products, F. Shahidi, Ed., vol. 3, pp. 175193, John Wiley \& Sons, 6th edition, 2005.

[2] K. Alexiadou and N. Katsilambros, "Nuts: anti-atherogenic food?" European Journal of Internal Medicine, vol. 22, no. 2, pp. 141-146, 2011.

[3] J. Yang, R. H. Liu, and L. Halim, "Antioxidant and antiproliferative activities of common edible nut seeds," LWT-Food Science and Technology, vol. 42, no. 1, pp. 1-8, 2009.

[4] J. Sabate, G. E. Fraser, K. Burke, S. F. Knutsen, H. Bennett, and K. D. Lindsted, "Effects of walnuts on serum lipid levels and blood pressure in normal men," The New England Journal of Medicine, vol. 328, no. 9, pp. 603-607, 1993.

[5] D. Zambón, J. Sabaté, S. Muñoz et al., "Substituting walnuts for monounsaturated fat improves the serum lipid profile of hypercholesterolemic men and women. A randomized crossover trial," Annals of Internal Medicine, vol. 132, no. 7, pp. 538-546, 2000.

[6] B. Muthaiyah, M. M. Essa, M. Lee, V. Chauhan, K. Kaur, and A. Chauhan, "Dietary supplementation of walnuts improves memory deficits and learning skills in transgenic mouse model of Alzheimer's disease," Journal of Alzheimer's Disease, vol. 42, no. 4, pp. 1397-1405, 2014.

[7] U. Gecgel, T. Gumus, M. Tasan, O. Daglioglu, and M. Arici, "Determination of fatty acid composition of gamma-irradiated hazelnuts, walnuts, almonds, and pistachios," Radiation Physics and Chemistry, vol. 80, no. 4, pp. 578-581, 2011.

[8] Joint FAO/IAEA/WHO Expert Committee, WHO, Geneva, Switzerland, 1981.

[9] C. Ayda, B. I. Engin, M. Polat, and T. Aydin, "Electron spin resonance study of $\gamma$-irradiated Anatolian chickpea (Cicer arietinum L.)," Radiation Effects and Defects in Solids, vol. 163, no. 1, pp. 7-17, 2008.

[10] M. G. Sajilata and R. S. Singhal, "Effect of irradiation and storage on the antioxidative activity of cashew nuts," Radiation Physics and Chemistry, vol. 75, no. 2, pp. 297-300, 2006.

[11] F. Diehl, Effects of Combination Processes on the Nutritive Value of Food. Combination Processes in the Food Irradiation, International Atomic Agency, Vienna, Austria, 1981.

[12] V. Komolprasert, "Packaging for foods treated by ionizing radiation," in Packaging for Non Thermal Processing of Food, J. H. Han, Ed., pp. 87-116, IFT Press, Blackwell Publishing, 2007.

[13] K. E. Paquette, "Irradiation of prepackaged food: evaluation of the Food and Drug Administration's regulation of the packaging materials," in Irradiation of Food and Packaging: Recent Developments, V. Komolprasert and K. Morehouse, Eds., vol. 875 of ACS Symposium Series, pp. 182-202, Oxford Press, 2004.

[14] V. J. Sinanoglou, K. Kokkotou, C. Fotakis, I. Strati, C. Proestos, and P. Zoumpoulakis, "Monitoring the quality of $\gamma$-irradiated macadamia nuts based on lipid profile analysis and Chemometrics. Traceability models of irradiated samples," Food Research International, vol. 60, pp. 38-47, 2014.

[15] P. Zoumpoulakis, V. J. Sinanoglou, A. Batrinou, I. F. Strati, S. Miniadis-Meimaroglou, and K. Sflomos, "A combined methodology to detect $\gamma$-irradiated white sesame seeds and evaluate the effects on fat content, physicochemical properties and protein allergenicity," Food Chemistry, vol. 131, no. 2, pp. 713-721, 2012.

[16] AOAC, Official Methods of Analysis, Association of Official Analytical Chemists, Arlington, Va, USA, 15th edition, 1990.

[17] H. Egli, Kjeldahl Method, BÜCHI Labortechnik AG, Flawil, Switzerland, 2nd edition, 2008.

[18] V. J. Sinanoglou and S. Miniadis-Meimaroglou, "Fatty acid of neutral and polar lipids of (edible) Mediterranean cephalopods," Food Research International, vol. 31, no. 6-7, pp. 467-473, 1998.

[19] V. J. Sinanoglou, I. F. Strati, S. M. Bratakos, C. Proestos, P. Zoumpoulakis, and S. Miniadis-Meimaroglou, "On the combined application of Iatroscan TLC-FID and GC-FID to identify total, neutral, and polar lipids and their fatty acids extracted from food," ISRN Chromatography, vol. 2013, Article ID 859024, 8 pages, 2013.

[20] C. Fotakis, D. Christodouleas, K. Kokkotou et al., "NMR metabolite profiling of Greek grape marc spirits," Food Chemistry, vol. 138, no. 2-3, pp. 1837-1846, 2013.

[21] Y. Ma, X. Lu, X. Liu, and H. Ma, "Effect of ${ }^{60}$ Coy-irradiation doses on nutrients and sensory quality of fresh walnuts during storage," Postharvest Biology and Technology, vol. 84, pp. 36-42, 2013.

[22] M. Al-Bachir, "Effect of gamma irradiation on fungal load, chemical and sensory characteristics of walnuts (Juglans regia L.)," Journal of Stored Products Research, vol. 40, no. 4, pp. 355362, 2004.

[23] P. Sánchez-Bel, I. Egea, F. Romojaro, and M. C. MartínezMadrid, "Sensorial and chemical quality of electron beam irradiated almonds (Prunus amygdalus)," LWT_Food Science and Technology, vol. 41, no. 3, pp. 442-449, 2008. 
[24] R. Bhat, K. R. Sridhar, C. C. Young, A. A. Bhagwath, and S. Ganesh, "Composition and functional properties of raw and electron beam-irradiated Mucuna pruriens seeds," International Journal of Food Science and Technology, vol. 43, no. 8, pp. 13381351, 2008.

[25] F. Khallouki, L. Mannina, S. Viel, and R. W. Owen, “Thermal stability and long-chain fatty acid positional distribution on glycerol of argan oil," Food Chemistry, vol. 110, no. 1, pp. 57-61, 2008. 

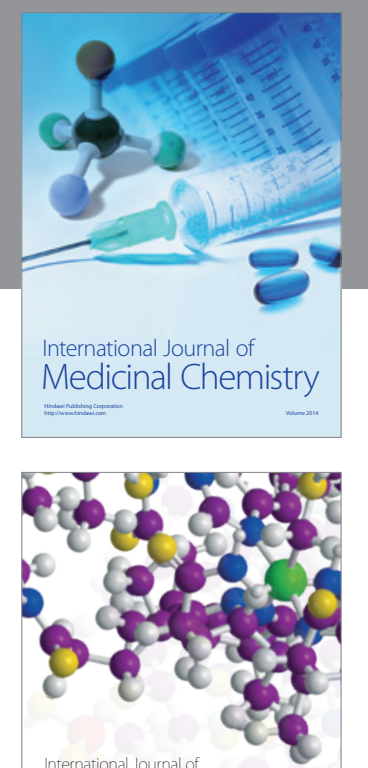

\section{Carbohydrate} Chemistry

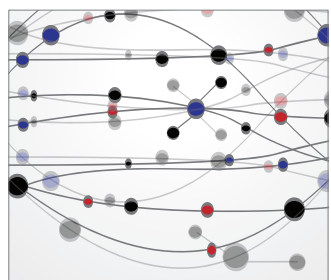

The Scientific World Journal
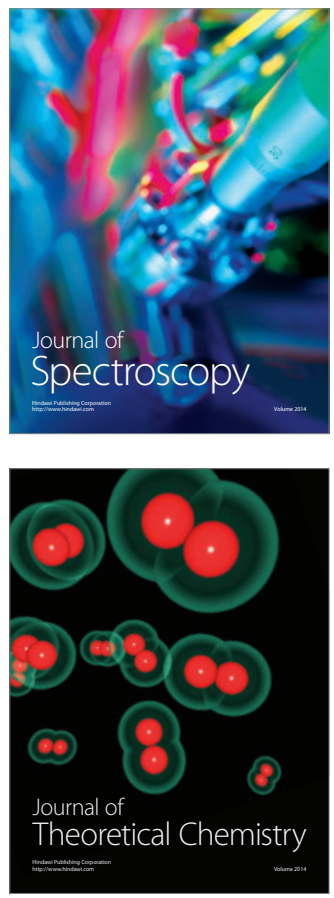
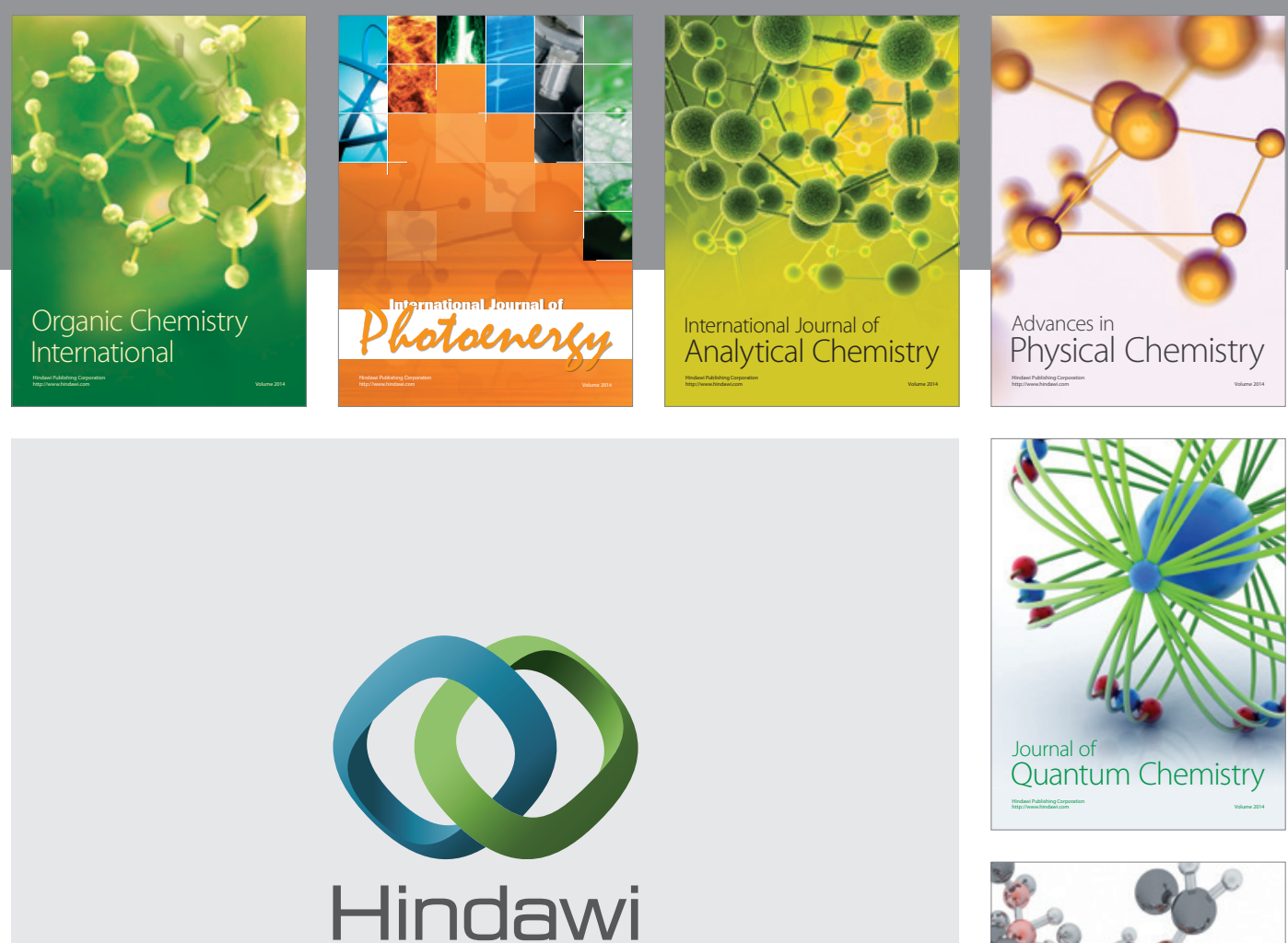

Submit your manuscripts at

http://www.hindawi.com

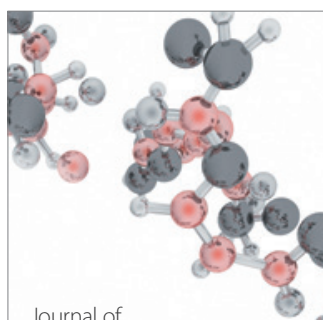

Analytical Methods

in Chemistry

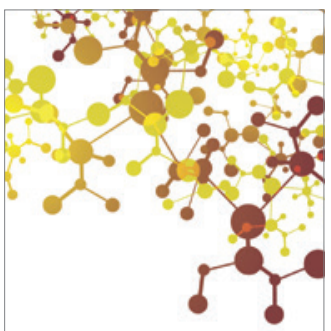

Journal of

Applied Chemistry

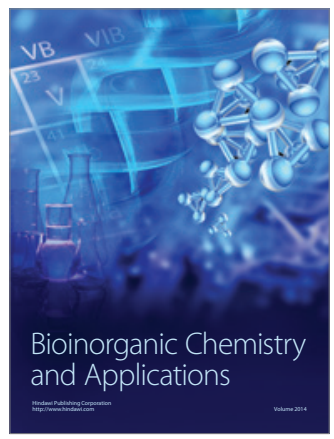

Inorganic Chemistry
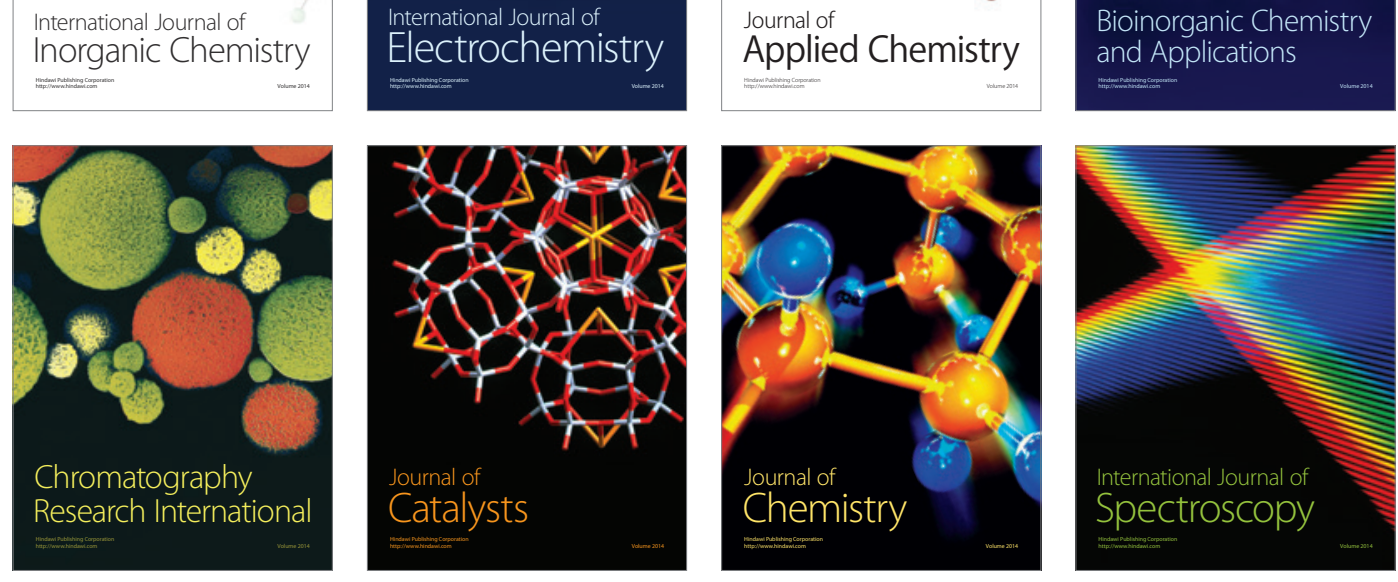